\title{
Alterações oculares induzidas pela quimioterapia
}

\author{
Ocularchanges induced bychemotherapy
}

\author{
Alessandra Pinheiro Chaves ${ }^{1}$ \\ José Álvaro Pereira Gomes ${ }^{2}$ \\ Ana Luisa Höfling-Lima ${ }^{3}$
}

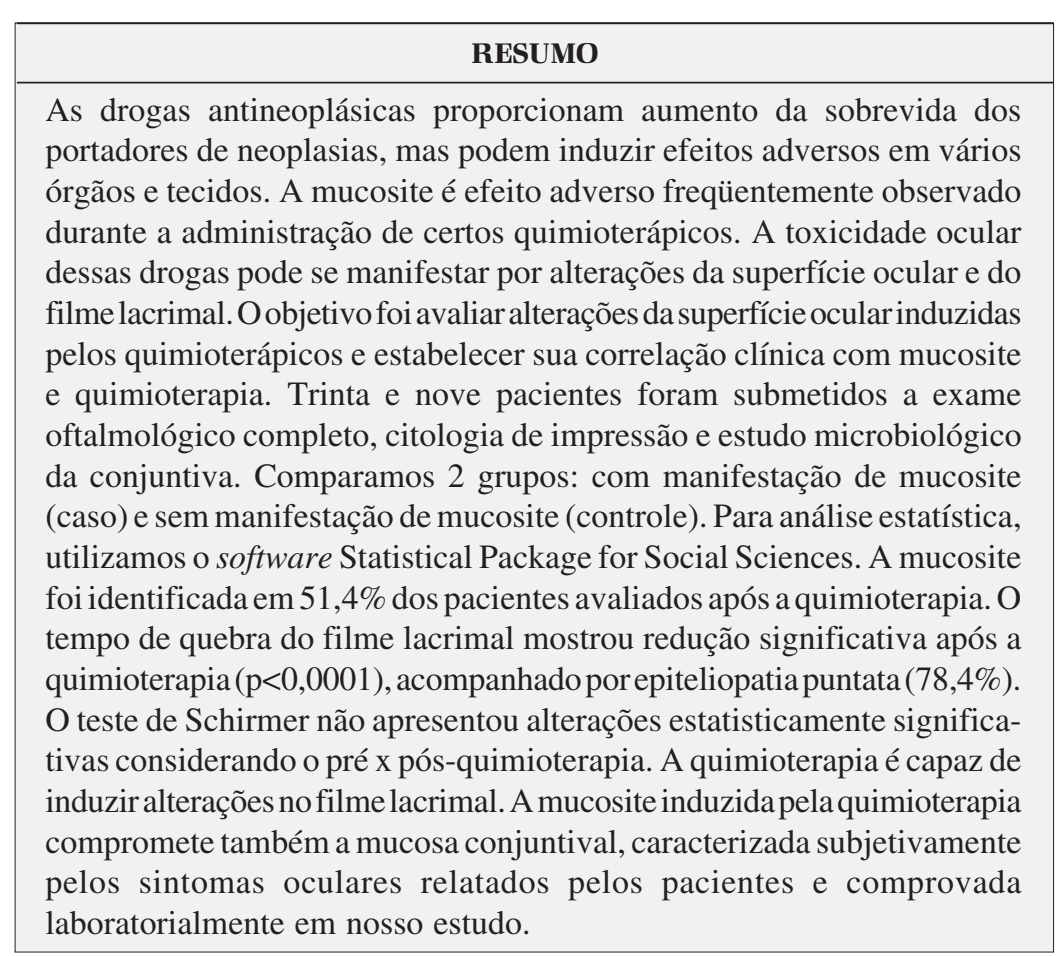

Descritores: Quimioterapia/efeitos adversos; Mucosite/induzida quimicamente; Neoplasias/ quimioterapia; Conjuntiva/citologia

\section{INTRODUÇÃO}

O uso das drogas antineoplásicas tem proporcionado aumento da sobrevida dos pacientes portadores de neoplasias diversas. Sabe-se, no entanto, que a exposição mais prolongada a essas drogas aumenta o risco de efeitos colaterais em diferentes órgãos e tecidos ${ }^{(1-2)}$.

Os quimioterápicos atuam sobre as células normais e as neoplásicas em diferentes proporções devido às diferenças quantitativas entre os processos metabólicos destas duas populações celulares. Uma das peculiaridades das células tumorais é a sua alta taxa de divisão celular. As células da medula óssea, dos folículos pilosos e de revestimento das mucosas também têm alta taxa de proliferação celular. Desta maneira, podemos entender alguns dos efeitos tóxicos associados aos quimioterápicos, tais como mielossupressão, alopécia e mucosite ${ }^{(3-5)}$.

A mucosite é um dos efeitos adversos mais freqüentes e limitantes observados durante a administração de certos quimioterápicos, como 5-fluorouracil (5-FU), metotrexato, doxorrubicina, bem como nos regimes de quimioradioterapia concomitante (QRT). Pacientes tratados com combinações específicas de certas drogas são ainda mais susceptíveis ao envolvimento 
da mucosa, causando sua inflamação e ulceração, o que predisporia ao desenvolvimento de infecção secundária em uma grande proporção de pacientes ${ }^{(6)}$. De acordo com o "Common Toxicity Criteria" (CTC), a mucosite pode ser classificada de acordo com a superfície mucosa acometida (faringite, estomatite, colite, esofagite, gastrite, vaginite, proctite $)^{(7)}$. Os sintomas iniciais descritos pelos pacientes na vigência de mucosite são sensação de queimação na cavidade oral, mas qualquer superfície mucosa pode apresentar eritema e progredir para erosão e ulceração durante a quimioterapia $(\mathrm{QT})^{(8)}$.

A superfície ocular é constituída pelos epitélios da córnea, limbo e o epitélio mucoso da conjuntiva. Suas funções principais são: 1) formar uma barreira de proteção à perda líquida e evitar a entrada de patógenos; 2) juntamente com o filme lacrimal, compor uma superfície lisa e regular e 3) possuir mecanismos cicatriciais altamente desenvolvidos para responder às agressões. Histologicamente estes epitélios são do tipo pavimentoso, estratificado, não queratinizado, dotados de uma membrana basal composta basicamente por colágeno tipo IV e proteínas da matriz extracelular. O epitélio conjuntival ainda apresenta células caliciformes que formam o componente de mucina do filme lacrimal, fundamental para sua estabilidade e interação com a superfície ocular ${ }^{(9-10)}$.

A ceratoconjuntivite sicca pode resultar de deficiência lacrimal por alterações das camadas de mucina, aquosa ou lipídica do filme lacrimal. As provas que determinam diretamente a deficiência aquosa do filme lacrimal são quantitativas, sendo o teste de Schirmer o mais utilizado. As provas capazes de identificar a deficiência de mucina são o tempo de ruptura do filme lacrimal (BUT), a coloração pelo rosa bengala, a prova de cristalização da lágrima e a citologia de impressão $(\mathrm{CI})^{(11)}$. A CI foi inicialmente descrita em $1977^{(12)}$ como uma técnica não invasiva, capaz de reproduzir achados compatíveis com uma biópsia superficial da conjuntiva. Com a CI é possível avaliar a morfologia e o grau de metaplasia do epitélio da superfície ocular e, com base na aparência das células epiteliais e na densidade das células caliciformes da conjuntiva, é possível classificar o grau de comprometimento da superfície ocular ${ }^{(13-14)}$.

De acordo com dados do "National Registry of Drug-Induced Ocular Side Effects", a toxicidade ocular das drogas antineoplásicas pode se manifestar por alterações da superfície ocular e do filme lacrimal, tais como ceratoconjuntivite sicca, conjuntivite, blefarite, ceratite, opacidade da córnea, além de ectrópio cicatricial, edema periorbitário, estenose canalicular, ptose, paralisia dos músculos extra-oculares, diplopia, distúrbios visuais, catarata, retinopatia e atrofia óptica ${ }^{(1,15-39)}$.

Este estudo tem como objetivo avaliar as alterações da superfície ocular induzidas pela QT em pacientes portadores de neoplasias e estabelecer a correlação clínica entre a QT, mucosite e as alterações da superfície ocular.

\section{MÉTODOS}

Para este estudo, foram selecionados 39 pacientes, sendo
35 do ambulatório de quimioterapia da Escola Paulista de Medicina e 4 do Hospital Sírio Libanês. Os pacientes apresentavam idade entre 23 e 74 anos $(53,8 \pm 13,7$ anos), sendo 17 homens $(43,6 \%)$ e 22 mulheres $(56,4 \%)$, classificados conforme a etnia nos seguintes grupos: amarelos $(7,7 \%)$, brancos $(56,4 \%)$, negros $(10,3 \%)$ e pardos $(25,6 \%)$. Dois dos pacientes foram excluídos do estudo por apresentarem comprometimento sistêmico grave após a QT.

Os critérios de inclusão adotados foram a presença de neoplasia com indicação de quimioterapia isolada ou associada à radioterapia em pacientes virgens de tratamento e "performance status" 0, 1 ou 2 pela ECOG (Eastern Cooperative Oncology Group $)^{(7,40)}$. Adotamos como critérios de exclusão do estudo pacientes com sobrevida inferior a 3 meses, HIV positivos, usuários de lentes de contato, diabéticos, portadores de doenças reumatológicas, pacientes com diagnóstico prévio de olho seco ou em uso de medicações tópicas ou sistêmicas que pudessem afetar a estabilidade do filme lacrimal.

Para análise dos dados, comparamos um grupo de pacientes com manifestações de mucosite com outro grupo sem manifestação de mucosite (controle).

Após a obtenção do termo de consentimento devidamente assinado por cada paciente ou pelo seu responsável, foram realizados os seguintes exames: medida da AV para perto e longe utilizando as tabelas de Snellen e Jaeger (com e sem correção), ectoscopia, BUT, biomicroscopia, teste de Schirmer, tonometria de aplanação (tonômetro de Goldmann), refração dinâmica, refração estática, mapeamento da retina, CI, citologia e coleta de material dos fórnices conjuntivais para estudo microbiológico. Tais exames diagnósticos foram realizados nesta ordem com a finalidade de avaliar a estabilidade do filme lacrimal e a sua camada aquosa, bem como avaliar possíveis alterações da superfície ocular antes do tratamento quimioterápico, sendo repetido após o mesmo (intervalo médio de 13,1 dias). O BUT foi avaliado após a instilação de uma gota de Fluoresceína (Lab. Allergan Frumtost, São Paulo, Brasil) no fórnice conjuntival, solicitando ao paciente que piscasse 2 a 3 vezes para distribuir o corante uniformemente. $\mathrm{O}$ paciente permaneceu com os olhos abertos sem piscar por alguns segundos e foi observado à lâmpada de fenda o tempo entre o último piscar e o aparecimento do primeiro ponto de quebra do filme lacrimal ao exame com luz azul de cobalto, sem que o examinador tocasse as suas pálpebras ou houvesse abertura excessiva da fenda palpebral. Foram consideradas as médias obtidas em 3 medidas consecutivas. Para o teste de Schirmer I, procedeu-se à instilação de proximetacaína $0,5 \%$ (Anestalcon ${ }^{\circledR}$ Lab. Alcon, São Paulo, Brasil) no fundo de saco conjuntival $1 \mathrm{~min}$ antes de realizar o teste, seguida pela secagem do mesmo antes de realizar as medidas. Para a realização deste exame, utilizou-se fitas de papel filtro de Whatman $\mathrm{n}^{\circ} 41$ de $5 \mathrm{~mm}$ de largura por $35 \mathrm{~mm}$ de comprimento, colocando-as na junção dos $2 / 3$ mediais com o terço lateral da pálpebra inferior de cada olho por 5 minutos. Após a retirada dos filtros, os mesmos tinham a parte úmida medida.

Para avaliação do padrão da coloração pela fluoresceína, 
examinou-se a conjuntiva bulbar e a córnea à biomicroscopia, quantificando a epiteliopatia ponteada em escores para cada quadrante da córnea e da conjuntiva, considerando como escore final a média obtida nos 4 quadrantes ( 0 - nenhum, 1 leve, 2 - moderado e 3 - severo).

Para análise dos resultados deste estudo, classificamos como olho seco aqueles olhos que apresentavam pelo menos 2 dos 3 critérios seguintes: Teste de Schirmer (anormal se $<5 \mathrm{~mm}$ 15 min), BUT (anormal se $<5$ segundos) e padrão fluoresceínico anormal se $\geq 1$.

O material para o exame microbiológico foi colhido no momento da consulta, semeado em placas de ágar sangue, chocolate e Sabouraud, além de 2 lâminas, sendo uma fixada em metanol para realização do Giemsa e outra fixada pelo calor para realização do Gram. O material foi enviado para ser processado no laboratório de microbiologia ocular do Departamento de Oftalmologia da UNIFESP - EPM.

As amostras para CI foram coletadas ao final da consulta, utilizando discos de acetato de celulose (Millipore Corporation, código de catálogo HAWP304F0, com poro de $0,45 \mu \mathrm{m}$ ), confeccionados com trépanos corneanos de 7,0 $\mathrm{mm}$ de diâmetro e esterelizados em óxido de etileno, sem contato direto dos dedos para prevenir a adesão de células da polpa digital na sua superfície. O disco de papel filtro era pressionado no quadrante temporal superior da conjuntiva bulbar (a 3-4 $\mathrm{mm}$ do limbo) em ambos os olhos do paciente com uma pinça de extremidade aplanada, por um período de 5 segundos, mantendo pressão suave e uniforme em toda sua extensão para garantir uma boa colheita de células. Em seguida, o papel era cuidadosamente removido com a mesma pinça e posicionado sobre uma lâmina limpa, seguida pela fixação imediata por álcool a 95\%, para posterior coloração e montagem das lâminas. A técnica de coloração utilizada para este estudo baseou-se na técnica de citologia de impressão de Martinez et al. ${ }^{(41)}$ que usa ácido periódico de Schiff (PAS), hematoxilina e Papanicolaou modificado, com algumas alterações. A CI foi avaliada conforme classificação proposta por Nelson ${ }^{(14)}$, variando de 0 a 3 conforme os achados citológicos descritos no quadro 1 .

Para a análise estatística, realizou-se análise exploratória dos dados, utilizando proporções para as variáveis categóricas e médias \pm desvios-padrão para variáveis escalares, além das análises gráficas para determinação dos tipos de parâmetros alcançados na amostra. Posteriormente, as variáveis escalares foram comparadas pelo do teste de Mann-Whitney e as variá- veis categóricas foram comparadas pelo teste do qui-quadrado ou teste exato de Fisher, quando necessário. Para a comparação em dois momentos utilizou-se o teste de McNemar.

Todos os cálculos foram realizados com o auxílio do software SPSS (Statistical Package for Social Sciences), versão 11.0, sendo considerados como estatisticamente significantes valores bicaudais de $\mathrm{p}$ inferiores a $5 \%(\mathrm{p}<0,05)$.

\section{RESULTADOS}

Para melhor análise dos dados, os pacientes foram agrupados conforme a doença de base em 4 grupos, de acordo com o tipo de tumor. Os tumores do trato genito-urinário (TGU) representaram 25,6\% dos casos, do trato gastro-intestinal (TGI) 25,6\%, do trato respiratório (TR) 17,9\% e outros tumores em 30,8\%.

A avaliação das drogas foi baseada no mecanismo de ação das mesmas, classificando-as em: agentes alquilantes (G1) utilizadas por 27 pacientes (69,2\%), antimetabólitos (G2) usadas em 20 pacientes $(51,3 \%)$, antibióticos (G3) em 13 pacientes $(33,3 \%)$ e antimitóticos (G4) em 10 pacientes $(25,6 \%)$.

A monoterapia foi utilizada em apenas $25,6 \%$ dos pacientes, enquanto que $74,4 \%$ utilizaram associação de grupos de medicações. Em 28,2\% as associações foram entre drogas de apenas um grupo, em $48,7 \%$ drogas de dois grupos e $23,1 \%$ com drogas de três grupos diferentes. A radioterapia foi realizada em $41,0 \%$ dos pacientes.

A mucosite foi identificada em 51,4\% dos 37 pacientes avaliados após a quimioterapia. A avaliação pós QT foi realizada entre os dias 7 e 27 após o primeiro ciclo da quimioterapia (média 13,1d), sendo realizada no D 14 em 37,8\% dos pacientes e em $91,9 \%$ dos pacientes até o D 17.

\section{Comparação entre pacientes antes e após $Q T$}

A acuidade visual sem correção (AVS/C) antes da QT variou de movimento de mãos (MM) a 20/20 em ambos os olhos, não havendo variação significativa após a QT. A PIO não mostrou variação significativa, comparando-se os seus resultados antes e após a QT, apresentando médias de 11,8 (dp $\pm 2,4 \mathrm{mmHg})$ e 12,4 $(\mathrm{dp} \pm 2,3 \mathrm{mmHg})$, respectivamente.

Quatorze pacientes $(37,8 \%)$ apresentaram queixas oculares após a QT (12 deles com mucosite). As queixas relatadas foram: ardor ocular $(45,9 \%)$, hiperemia conjuntival $(18,9 \%)$ e embaçamento visual $(18,9 \%)$. Dos pacientes submetidos a RT,

\begin{tabular}{|c|c|c|c|c|}
\hline \multicolumn{5}{|c|}{ Quadro 1. Microscopia } \\
\hline Grau Cl & Células epiteliais & Citoplasma & Núcleo/N:C & Células caliciformes \\
\hline 0 & Pequenas/arredondadas & Eosinofílico & Grande e basofílico/1:2 & >500 células $/ \mathrm{mm}^{2}$ \\
\hline 1 & Ligeiramente aumentadas/poligonais & Eosinofílico & Menor/1:3 & (350-500 células $\left./ \mathrm{mm}^{2}\right)$ \\
\hline 2 & $\begin{array}{l}\text { Aumentadas/poligonais/ } \\
\text { ocasionalmente multinucleadas }\end{array}$ & Coloração variável & Pequenos/1:4-1:5 & (100-350células $\left./ \mathrm{mm}^{2}\right)$ \\
\hline \multirow[t]{2}{*}{3} & Grandes/poligonais & Basofílico & Pequeno, picnótico ou & (<100 células $\left./ \mathrm{mm}^{2}\right)$ \\
\hline & & & mesmo ausente/1:6 & \\
\hline
\end{tabular}


observou-se ardor em 50\%, hiperemia em 18,8\%, embaçamento visual em $18,8 \%$.

Seguindo definição utilizada nesse trabalho, o olho seco foi diagnosticado em 10,3\% dos pacientes antes da QT, sendo identificado em 62,2\% dos casos após a QT. Dos pacientes sem olho seco antes da QT, 60,6\% apresentavam olho seco após a QT $(\mathrm{p}<0,001)$.

A epiteliopatia ponteada esteve presente em $15,4 \%$ dos olhos examinados antes da QT, passando para 78,4\% após a QT. Dentre os olhos sem epiteliopatia prévia, 74,2\% passaram a apresentar este achado após a QT, elevando esta taxa significativamente $(\mathrm{p}<0,001)$.

Comparando os resultados do teste de Schirmer antes e após a QT, observou-se que em 20,5\% dos olhos o teste de Schirmer foi $<5 \mathrm{~mm}$ no pré QT, enquanto apenas $13,5 \%$ destes apresentavam valores inferiores a $5 \mathrm{~mm}$ no pós QT (Figura 1). Dentre os pacientes com resultado normal antes da QT, 6,9\% apresentaram resultados inferiores a $5 \mathrm{~mm} / 5 \mathrm{~min}$ após a QT. Paradoxalmente, daqueles pacientes com resultado anormal antes da QT, 37,5\% mantiveram olho seco e 62,5\% passaram a apresentar resultados normais após a QT. Sua média foi de $15,3 \mathrm{~mm}(\mathrm{dp} \pm 12,6)$ no pré-QT e 15,0 mm (dp $\pm 9,7)$ no pós QT, não sendo observada diferença estatisticamente significativa.

A taxa de olho seco baseado no BUT $(<5 \mathrm{seg})$ variou de $25,6 \%$ para $64,9 \%$ após a QT (p<0,0001) (Figura 1). Dos 27 pacientes com BUT normal antes da QT, apenas $48,1 \%$ apresentaram BUT normal após a QT. Aqueles com BUT compatível com olho seco antes da QT mantiveram-se na mesma classificação após a QT em $100 \%$ dos casos.

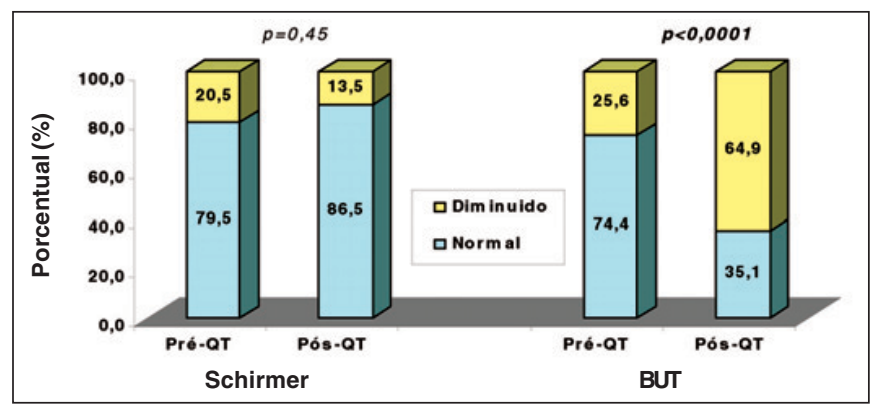

Figura 1 - Classificação de olho seco de acordo com o teste de Schirmer e BUT

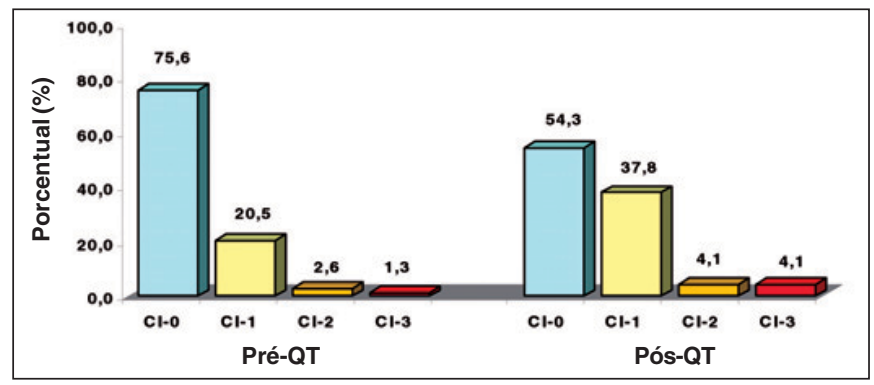

Figura 2 - Classificação da citologia de impressão por olhos estudados
A CI antes da QT foi classificada em classes 0, 1, 2 e 3, estando 59 dos 78 olhos $(75,6 \%)$ na classe $0,16(20,5 \%)$ na classe $1,2(2,6 \%)$ na classe 2 e $1(1,3 \%)$ na classe 3 . Dezenove olhos $(17,9 \%)$ apresentavam muco e $100 \%$ deles apresentava células epiteliais degeneradas e em degeneração à análise citológica. A CI após a QT apresentava 40 dos 74 olhos $(54,3 \%)$ na classe $0,28(37,8 \%)$ na classe $1,3(4,1 \%)$ na classe 2 e $3(4,1 \%)$ na classe 3 (Figura 2$)$. Nove olhos $(12,2 \%)$ apresentavam muco, $100 \%$ deles apresentava células epiteliais degeneradas e em degeneração e 5 olhos apresentavam bacilos Gram+ à análise microbiológica, identificados como Corinebacterium xerosis à cultura.

A CI pós-QT evidenciou hipertrofia das células do epitélio conjuntival em $67,6 \%$ dos casos e metaplasia em $35,1 \%$ dos casos. Dos pacientes submetidos a RT, observou-se hipertrofia em $62,5 \%$ e metaplasia em $31,3 \%$ dos casos.

\section{Comparação entre pacientes com mucosite $x$ sem mucosite após $Q T$}

Dentre os 19 pacientes com mucosite, $63,2 \%$ apresentavam queixas oculares contra apenas $11,1 \%$ dos pacientes sem mucosite $(\mathrm{p}=0,002)$. As queixas oculares foram caracterizadas por ardor ocular em $73,3 \%$, hiperemia conjuntival em $26,3 \%$ e embaçamento em $31,6 \%$ dos casos, enquanto no grupo controle estas queixas oculares foram $16,7 \%, 11,1 \%$ e 5,6\% respectivamente (Figura 3).

Em relação aos achados da CI, observamos que a hipertrofia foi observada em $72,2 \%$ dos que não apresentaram mucosite e em $63,2 \%$ daqueles que a desenvolveram. Já a metaplasia foi observada em $27,8 \%$ dentre os que não desenvolveram mucosite e em $42,1 \%$ dos casos com mucosite.

Os dados da CI pós-QT quanto à mucosite, podem ser comparados na tabela 1 .

\section{Comparação entre pacientes que fizeram uso monoterapia $x$ politerapia}

Dentre os pacientes que não utilizaram associação de drogas, 9,1\% apresentavam critérios diagnósticos de olho seco antes da QT, enquanto que após a QT este número aumentou para $77,8 \%$. Dentre os que associaram 2 grupos de drogas, estes número foram $15,8 \%$ e $52,6 \%$ e nos que associaram 3

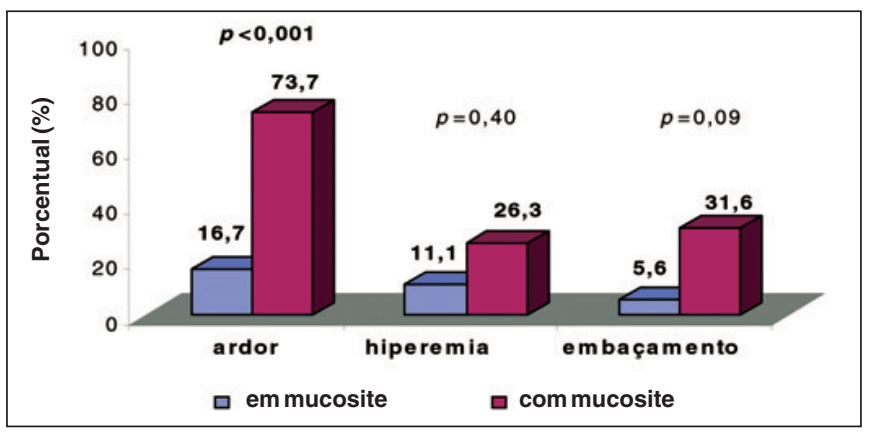

Figura 3 - Mucosite $\mathrm{x}$ sintomas oculares 
grupos de drogas $11,1 \%$ e $66,7 \%$ respectivamente. A mucosite foi observada em $55,6 \%$ dos pacientes que não fizeram associação de grupos de drogas, $57,9 \%$ de pacientes que associaram 2 grupos de drogas e em $33,3 \%$ dos pacientes que associaram 3 grupos de drogas, enquanto as queixas oculares foram relatadas em $22,2 \%, 52,6 \%$ e $22,2 \%$ respectivamente.

Dos pacientes que utilizaram associação de grupos de drogas, $10,3 \%$ preenchiam critérios diagnósticos de olho seco antes da QT, aumentando para 58,6\% após a QT ( $\mathrm{p}=0,001)$. A mucosite foi observada em $14(48,3 \%)$ e as queixas oculares em $12(41,4 \%)$ deste pacientes (ambas sem significância estatística) (Tabela 2). Dentre aqueles submetidos a radioterapia, o olho seco foi diagnosticado antes da QT em apenas 6,3\% dos pacientes, enquanto após a QT este número aumentou para $68,8 \%(\mathrm{p}=0,02)$. A mucosite foi observada em $43,8 \%$ e as queixas oculares em $31,3 \%$ dos pacientes do mesmo grupo $(\mathrm{p}=0,42$ e $\mathrm{p}=0,47$ respectivamente). A tabela 3 compara as queixas relatadas pelos pacientes e os achados à CI nos diferentes grupos de drogas.

\section{DISCUSSÃO}

Na literatura médica, há relatos de mucosite e alterações oculares induzidas por diferentes quimioterápicos disponíveis na prática médica, tais como irritação ocular grave, depósitos corneais, incapacidade visual temporária durante o tratamento quimioterápico ${ }^{(42)}$, edema periorbitário e epífora ${ }^{(43)}$. Tullo el al. ${ }^{(44)}$ observaram alterações oftalmológicas transitórias durante a administração de gefitinibe. Devido a carência de trabalhos que avaliassem de maneira mais objetiva as altera-

\begin{tabular}{|lcccc|}
\hline \multicolumn{4}{|c|}{ Tabela } & 1. Mucosite $\mathbf{x}$ citologia de impressão pós quimioterapia \\
Mucosite & 0 & 1 & 2 & 3 \\
Não & $55,6 \%$ & $38,9 \%$ & $5,6 \%$ & - \\
Sim & $47,4 \%$ & $42,1 \%$ & - & $10,5 \%$ \\
Cl= citologia de impressão; QT= quimioterapia & \\
\hline
\end{tabular}

ções da superfície ocular e do filme lacrimal secundárias a QT, resolvemos analisar os resultados do teste de Schirmer, coloração de fluoresceína, BUT e CI nesses pacientes.

Turhal et al. avaliaram 31 pacientes, com diagnóstico de tumores do TGI (12), leucemias (9), linfomas (2), melanoma (2) e outros tumores (6), que apresentaram mucosite após a quimioterapia. A duração média da mucosite foi de 7,9 dias (3-23 dias). Os autores dividiram os pacientes em 2 grupos: 1) TU sólidos e 2) leucemias, observando que no grupo 1 a duração da mucosite e a sua gravidade foram maiores ${ }^{(45)}$. No presente estudo, os pacientes foram classificados em 4 grupos de tumores e a avaliação pós-QT foi realizada entre os dias 7 e 27 após o primeiro ciclo da quimioterapia, com intervalo médio de 13,1 dias. A mucosite foi identificada em 51,4\% dos 37 pacientes avaliados após a QT, ocorrendo em $40 \%$ dos pacientes com TU TGU, 50\% dos pacientes com TU TR, 66,7\% dos pacientes com TU TGI e 50\% dos pacientes com outros TU. Sessenta e três por cento dos pacientes com mucosite apresentavam queixas oculares contra apenas $11,1 \%$ dos pacientes sem mucosite $(\mathrm{p}=0,002)$.

O exame oftalmológico por nós realizado demonstrou que a acuidade visual sem e com correção, bem como a medida de pressão intra-ocular, não apresentaram nenhuma alteração após a QT. O BUT mostrou uma redução estatisticamente significativa após a QT ( $\mathrm{p}<0,0001)$, acompanhado por epiteliopatia puntata. Os resultados do teste de Schirmer apontam para uma elevação paradoxal no pós-QT em 62,5\% dos pacientes com Schirmer inferior a $5 \mathrm{~mm} / 5 \mathrm{~min}$, embora sua média dos valores absolutos no pré-QT x pós-QT não tenha evidenciado diferença estatisticamente significativa. Pode-se inferir que a QT induz alterações no componente de mucina do filme lacrimal, o que se reflete pelas alterações do BUT e da coloração por fluoresceína.

Em outro estudo ${ }^{(21)}$ foram avaliados 72 pacientes com diferentes neoplasias, submetidos a um regime sequencial de quimioterapia com MTX/ 5-FU, evidenciando toxicidade hematológica, gastrointestinal e epitelial, incluindo estomatite, gastroenterite, vômitos e anorexia, além de conjuntivite em $6 \%$ dos pacientes estudados. Os dados evidenciaram que a to-

\begin{tabular}{|c|c|c|c|c|}
\hline & Olho seco (Pré-QT) & Olho seco (Pós-QT) & Mucosite & Queixas \\
\hline Monoterapia & 10,0 & 75,0 & 50,0 & 25,0 \\
\hline Politerapia & 10,3 & 58,6 & 48,3 & 41,4 \\
\hline \multicolumn{5}{|c|}{ Quantidade de grupos } \\
\hline 1 & 9,1 & 77,8 & 45,5 & 22,2 \\
\hline 2 & 10,5 & 52,6 & 57,9 & 52,6 \\
\hline 3 & 11,1 & 66,7 & 33,3 & 22,2 \\
\hline \multicolumn{5}{|c|}{ Quantidade de drogas } \\
\hline 1 & 14,3 & 80,0 & 28,6 & 20,0 \\
\hline 2 & 9,1 & 54,5 & 63,6 & 45,5 \\
\hline 3 & 10,0 & 70,0 & 30,0 & 30,0 \\
\hline 4 & - & - & - & - \\
\hline QT=quimioterap & & & & \\
\hline
\end{tabular}




\begin{tabular}{|lccccc|}
\hline \multicolumn{5}{|c}{ Tabela 3. Sintomas oculares e achados citológicos à citologia de impressão conforme grupos de drogas estudados } \\
Grupos de drogas & Ardor (\%) & Hiperemia (\%) & Embaçamento(\%) & Hipertrofia (\%) & Metaplasia (\%) \\
G1 & 53,8 & 26,9 & 19,2 & 69,2 & 26,2 \\
G2 & 42,1 & 10,5 & 10,5 & 52,6 & 36,8 \\
G3 & 46,2 & 23,1 & 23,1 & 69,2 & 23,1 \\
G4 & 50,0 & 20,0 & 40,0 & 80,0 & 30,0 \\
Cl= citologia de impressão & \multicolumn{7}{c}{} \\
\hline
\end{tabular}

xicidade epitelial foi mais freqüente com a associação destas 2 drogas que com a sua administração isolada. No nosso trabalho, o diagnóstico de olho seco seguindo os critérios adotados foram observados em $14,3 \%$ dos pacientes pré-QT que fizeram uso de apenas 1 droga, 9,1\% dos pacientes que associaram 2 drogas e em $10 \%$ dos pacientes que associaram 3 ou mais drogas, e em $80 \%, 54,5 \%$ e $70 \%$, respectivamente, no pósQT. Nossa impressão é que a QT, independentemente do número de drogas, representa o fator determinante do desenvolvimento do olho seco. Mais estudos são necessários para melhor avaliar se a dose ou o tipo de QT influencia no desenvolvimento do olho seco nesses pacientes.

Connor et al. propuseram critérios diagnósticos de olho seco com base na $\mathrm{CI}$, tais como diminuição no número das células caliciformes da conjuntiva, diminuição do número de células agrupadas (identifica-se geralmente células isoladas), aumento do tamanho da célula, alteração da relação núcleo-citoplasma (às custas do aumento do volume do citoplasma) ${ }^{(46)}$. Nelson relatou a avaliação de 20 pacientes em uso de lágrimas artificiais sem preservantes por 8 semanas utilizando os testes de Schirmer, BUT, rosa bengala e padrão de fluoresceína, bem como a osmolaridade do filme lacrimal e o padrão da $\mathrm{CI}^{(14)}$. O autor observou que a CI demonstrou ser extremamente útil no diagnóstico de vários estados de ceratoconjuntivite seca, que geralmente está associada a metaplasia da superfície ocular. À medida que a ceratoconjuntivite seca progride, há diminuição da contagem de células caliciformes da conjuntiva e as células epiteliais assumem um tamanho maior e forma poligonal. Células inflamatórias raramente são vistas à CI e, quando presentes, sugerem a presença de um componente inflamatório que pode ser secundário a toxicidade ou doença da superfície ocular. Nenhuma correlação entre o padrão de rosa bengala ou o teste de Schirmer e a CI foi estabelecida, sugerindo que a metaplasia escamosa pode ocorrer na presença de secreção lacrimal normal e que a melhora no padrão da coloração pelo rosa bengala, bem como a osmolaridade do filme lacrimal, não são acompanhados pela melhora do grau de metaplasia escamosa da conjuntiva. Tseng et al. ${ }^{(47)}$ sugeriram que a patogênese da metaplasia escamosa do epitélio da superfície ocular pode ser secundária à perda da vascularização e ao processo cicatricial. Os autores afirmaram ainda que a ausência de fatores circulantes que mantém a diferenciação epitelial ou a presença de outros fatores introduzida pelo processo inflamatório intenso na mucosa pode favorecer o surgimento de alterações epiteliais na superfície ocular. Em nosso estudo, a CI pré x pósQT evidenciou mudança na distribuição dos pacientes entre as diferentes classes, sendo notado aumento do percentual nas classes 1, 2 e 3, comprovando histologicamente que há envolvimento da mucosa conjuntival induzido pela QT. Além disso, a CI pós-QT evidenciou hipertrofia das células do epitélio conjuntival em $67,6 \%$ dos casos e metaplasia em $35,1 \%$ dos casos. A hipertrofia foi observada em $72,2 \%$ dos que não apresentaram mucosite e em $63,2 \%$ daqueles que a desenvolveram. Já a metaplasia foi observada em $27,8 \%$ dentre os que não desenvolveram mucosite e $42,1 \%$ dos casos de mucosite. Esses achados podem explicar as alterações encontradas nos testes que avaliam a estabilidade lacrimal (BUT e coloração por fluoresceína) determinada pelo componente de mucina, produzido principalmente pelas células caliciformes da conjuntiva ${ }^{(14)}$.

Stenson et al. ${ }^{(48)}$ avaliou laboratorialmente 852 casos de conjuntivite crônica por análise citológica e a cultura de Corynobacterium xerosis (que tipicamente acompanha queratinização do epitélio nos olhos secos) esteve presente em 120 pacientes. $\mathrm{O}$ achado citológico mais frequientemente observado foi degeneração das células epiteliais, sob a forma de picnose ou graus variados de queratinização, que podem ocorrer como efeito secundário de inflamação crônica, infecção e/ou representar afecção epitelial primária predispondo à irritabilidade conjuntival persistente. Os autores apontam o exame citológico da conjuntiva como um recurso diagnóstico útil nos casos de conjuntivites crônicas, toxicidade por drogas, alergias e olho seco. Antes da QT, observou-se que dezenove olhos $(17,9 \%)$ apresentavam muco e $100 \%$ deles apresentava células epiteliais degeneradas e em degeneração à análise citológica; após a QT, nove olhos (12,2\%) apresentavam muco, 100\% deles apresentava células epiteliais degeneradas e em degeneração e 5 olhos apresentavam bacilos Gram+ à análise microbiológica, identificados como Corinebacterium xerosis à cultura. Esse achado é mais um indicativo do desenvolvimento de olho seco em pacientes submetidos a QT

Em resumo, pode-se inferir que a QT é capaz de induzir alterações no filme lacrimal, comprovadas neste estudo pelo BUT, teste de Schirmer e biomicroscopia. A mucosite induzida pela QT compromete também a mucosa conjuntival, caracterizada subjetivamente pelos sintomas oculares relatados pelos pacientes e comprovada laboratorialmente pela CI e estudo microbiológico.

\section{ABSTRACT}

Antineoplastic agents offer an increase in the patients' survival rates, but may induce side effects in different organs and tis- 
sues. Mucositis is one of the most common side effects during chemotherapy with certain agents. The ocular toxicity induced by these drugs may manifest as changes in the ocular surface and in the tear film. The purpose of this research was to study the ocular surface changes induced by chemotherapy and to establish their clinical correlation with the chemotherapy and mucositis. The 39 selected patients underwent a full ophthalmological examination and supplementary tests. We compared 2 groups of patients: with (case) and without mucositis (control). Statistical analysis was performed with the Statistical Package for Social Sciences software. Mucositis was identified in $51.4 \%$ of the patients after chemotherapy. The brakeup time showed a statistically significant decrease after chemotherapy $(p<0.0001)$. Punctate epitheliopathy was seen in $78.4 \%$ of cases. Schirmer test results did not show significant changes pre $\mathrm{x}$ post-chemotherapy. Chemotherapy may induce tear film changes, as shown in this study by the brakeup time, Schirmer test and biomicroscopy. Chemotherapy-induced mucositis involves also the conjunctival mucosa, which was subjectively characterized by the ocular symptoms and proved in our study by impression cytology and microbiology.

Keywords: Chemotherapy/adverse effects; Drug therapy, mucositis/chemically induced; Neoplasms/drug therapy; Conjunctiva/cytology

\section{REFERÊNCIAS}

1. Fraunfelder FT, Meyer SM. Ocular toxicity of antineoplastic agents. Ophthalmology. 1983;90(1):1-3.

2. Loprinzi CL, Foote RL, Michalak J. Alleviation of cytotoxic therapy-induced normal tissue damage. Semin Oncol. 1995;22(2 Suppl 3):95-7.

3. Controle do câncer: uma proposta de integração ensino-serviço. Rio de Janeiro: Pró-Onco/NUTES; 1990.

4. Giglio AD. Modalidades terapêuticas para o tratamento do câncer. In: Giglio AD. Câncer: introdução ao seu estudo e tratamento. São Paulo: Pasqualin; 1996. p.38-58.

5. Boente P, Sampaio C, Del Giglio A. Agentes antineoplásicos. In: Silva P. Farmacologia. 5a ed. Rio de Janeiro: Guanabara-Koogan; 1998. cap. 112. p.1074-80.

6. Feld R. The role of surveillance cultures in patients likely to develop chemotherapy-induced mucositis. Support Care Cancer. 1997;5(5):371-5.

7. Common Toxicity Criteria Manual Version 2.0 (1999). March 1998, updated April 30, 1999. USA: National Cancer Institute; 1999.

8. Lembersky B, Posner M. Gastrointestinal toxicities. In: Kirkwood JM, Lotze MT, Yasko JM, editors. Current cancer therapeutics. 2nd ed. Philadelphia: Churchill Livingstone; 1996. cap.27. p.326-37.

9. Gomes JAP, Lima ALH, Adan CBD. Avaliação da superfície ocular. In: Lima ALH, Nishiwaki-Dantas MC, Ruiz-Alves M, editores. Manual do CBO: doenças externas oculares e córnea. Rio de Janeiro: Cultura Médica; 1999. v.1. p.57-109.

10. Kenyon KR. Anatomy and pathology of the ocular surface. Int Ophthalmol Clin. 1979;19(2):3-35.

11. Murube J. Ojo seco - dry eye. Madrid: Tecnimedia; 1997

12. Egbert PR, Lauber S, Maurice DM. A simple conjunctival biopsy. Am J Ophthalmol. 1977;84(6):798-801.

13. Dart J. Impression cytology of the ocular surface - research tool or routine clinical investigation? Br J Ophthalmol. 1997;81(11):930.

14. Nelson JD. Impression cytology. Cornea. 1988;7(1):71-81.

15. Sidi Y, Douer D, Pinkhas J. Sicca syndrome in a patient with toxic reaction to bussulfan. JAMA. 1977;238(18):1951.

16. Tannock IF, Boyd NF, DeBoer G, Erlichman C, Fine S, Larocque G, et al. A randomized trial of two dose levels of cyclophosphamide, methotrexate, and fluorouracil chemotherapy for patients with metastatic breast cancer. J Clin Oncol. 1988;6(9):1377-87.
17. Awidi AS. Blindness and vincristine. Ann Intern Med. 1980;93(5):781.

18. Laufman LR, Brenckman WD Jr, Stydnicki KA, Morgan ED, Collier M, Knick VB, et al. Clinical experience with leucovorin and 5-fluorouracil. Cancer. 1989;63(6 Suppl):1031-5.

19. Higa GM, Gockerman JP, Hunt AL, Jones MR, Horne BJ. The use of prophylactic eye drops during high-dose cytosine arabinoside therapy. Cancer. 1991;68(8):1691-3.

20. Climent MA, Ruiz A, Llombart-Cussac A, Fernandez-Martos C, Poveda A, Dorta $\mathrm{J}$ et al. Weekly docetaxel in patients with advanced malignancies. Toxicity profile and activity results [abstract]. Prog Am Soc Clin Oncol. 1999;18: 119a Abstract 453.

21. Bruckner HW, Cohen J. MTX/5-FU trials in gastrointestinal and other cancers. Semin Oncol. 1983;10(2 Suppl 2):32-9.

22. Madajewicz S, Petrelli N, Rustum YM, Campbell J, Herrera L, Mittelman A, et al. Phase I-II trial of high-dose calcium leucovorin and 5-fluorouracil in advanced colorectal cancer. Cancer Res. 1984;44(10):4667-9.

23. Rosenoff SH, Wolf ML, Chabner BA. Pseudomonas blepharoconjunctivitis: a complication of combination chemotherapy. Arch Ophthalmol. 1974;91(6):490-1.

24. Barletta JP, Fanous MM, Margo CE. Corneal and conjunctival toxicity with low-dose cytosine arabinoside. Am J Ophthalmol. 1992;113(5):587-8.

25. Hopen G, Mondino BJ, Johnson BL, Chervenick PA. Corneal toxicity with systemic cytarabine. Am J Ophthalmol. 1981;91(4):500-4.

26. Burstein HJ, Manola J, Younger J, Parker LM, Bunnell CA, Scheib R, et al. Docetaxel administered on a weekly basis for metastatic breast cancer. J Clin Oncol. 2000;18(6):1212-9.

27. Hamersley J, Luce JK, Florentz TR, Burkholder MM, Pepper JJ. Excessive lacrimation from fluorouracil treatment. JAMA. 1973;225(7):747-8.

28. Albert DM, Wong VG, Henderson ES. Ocular complications of vincristine therapy. Arch Ophthalmol. 1967;78(6):709-13.

29. Bixenman WW, Nicholls JV, Warwick OH. Oculomotor disturbances associated with 5-fluorouracil chemotherapy. Am J Ophthalmol. 1977;83(6):789-93.

30. Kende G, Sirkin SR, Thomas PR, Freeman AI. Blurring of vision: a previously undescribed complication of cyclophosphamide therapy. Cancer. 1979;44(1):69-71.

31. Miller DF, Bay JW, Lederman RJ, Purvis JD, Rogers LR, Tomsak RL. Ocular and orbital toxicity following intracarotid injection of BCNU (carmustine) and cisplatinum for malignant gliomas. Ophthalmology. 1985;92(3):402-6.

32. Breithaupt H, Pralle H, Eckhardt T, von Hattingberg M, Schick J, Löffler H. Clinical results and pharmacokinetics of high-dose cytosine arabinoside (HD ARA-C). Cancer. 1982;50(7):1248-57.

33. Ravindranathan MP, Paul VJ, Kuriakose ET. Cataract after busulphan treatment. Br Med J. 1972;1(5794):218-9.

34. Podos SM, Canellos GP. Lens changes in chronic granulocytic leukemia. Possible relationship to chemotherapy. Am J Ophthalmol. 1969;68(3):500-4.

35. McKeown CA, Swartz M, Blom J, Maggiano JM. Tamoxifen retinopathy. Br J Ophthalmol. 1981;65(3):177-9.

36. Kaiser-Kupfer MI, Kupfer C, Rodrigues MM. Tamoxifen retinopathy. A clinicopathologic report. Ophthalmology. 1981;88(1):89-93.

37. Shingleton BJ, Bienfang DC, Albert DM, Ensminger WD, Chandler WF, Greenberg HS. Ocular toxicity associated with high-dose carmustine. Arch Ophthalmol. 1982;100(11):1766-72.

38. Sanderson PA, Kuwabara T, Cogan DG. Optic neuropathy presumably caused by vincristine therapy. Am J Ophthalmol. 1976;81(2):146-50.

39. Fishman ML, Bean SC, Cogan DG. Optic atrophy following prophylactic chemotherapy and cranial radiation for acute lymphocytic leukemia. Am J Ophthalmol. 1976;82(4):571-6.

40. Bakemeier R, Qazi R. Basic concepts of cancer chemotherapy and principles of medical oncology. In: Rubin P, editor. Clinical oncology: a multidisciplinary approach for physicians and students. $7^{\text {th }}$ ed. Philadelphia: WB Saunders; 1993. cap. 8 p.105-16.

41. Martinez AJ, Mills MB, Jaceldo KB, Tio FO, Aigbivbalu IB, Hilsenbeck SB, Yee RW. Standardization of conjunctival impression cytology. Cornea. 1995;14(5):515-22.

42. Waikhom B, Fraunfelder FT, Henner WD. Severe ocular irritation and corneal deposits associated with capecitabine use. N Engl J Med. 2000;343(10):7401. Erratum in: N Engl J Med. 2000;343(19):1428. Walkhom B [corrected to Waikhom B].

43. Fraunfelder FW, Solomon J, Druker BJ, Esmaeli B, Kuyl J. Ocular sideeffects associated with imatinib mesylate (Gleevec). J Ocul Pharmacol Ther. 2003;19(4):371-5.

44. Tullo AB, Esmaeli B, Murray PI, Bristow E, Forsythe BJ, Faulkner K. Ocular findings in patients with solid tumours treated with the epidermal growth factor receptor tyrosine kinase inhibitor gefitinib ('Iressa', ZD1839) in Phase I and II clinical trials. Eye. 2005;19(7):729-38. 
45. Turhal NS, Erdal S, Karacay S. Efficacy of treatment to relieve mucositisinduced discomfort. Support Care Cancer. 2000;8(1):55-8.

46. Connor CG, Campbell JB, Tirey WW, Steel SA, Burke JH. Modification of impression cytology for in-office use. J Am Optom Assoc. 1991;62(12):898901.
47. Tseng SC, Hirst LW, Maumenee AE, Kenyon KR, Sun TT, Green WR. Possible mechanisms for the loss of goblet cells in mucin-deficient disorders. Ophthalmology. 1984;91(6):545-52.

48. Stenson S, Fedukowicz H, Newman R. Laboratory studies in chronic conjunctivitis. Ann Ophthalmol. 1983;15(12):1160-4. 